\title{
Conversion of Carbohydrates Under Microwave Heating
}

\author{
Aurore Richel and Michel Paquot
}

Additional information is available at the end of the chapter

http://dx.doi.org/10.5772/50628

\section{Introduction}

The non-energetic valorisation of renewable resources using efficient and eco-friendly methodologies is the central axis of the "green chemistry" concept. In particular, the chemical and chemo-enzymatical transformation of carbohydrates arising from the hydrolysis of nonedible vegetal feedstock (i.e., lignocellulosic biomass) is a widely explored thematic for the production of new high-added value materials, synthons, and platform chemicals (Bozell, 2010). The use of mono-, di- and polysaccharides for the production of new chemicals constitutes thus a subject of special relevance from both academic and industrial points of view (Lichtenthaler, 2004). Amongst the 12 principles defining this "green chemistry" concept, the development of new effective synthetic protocols, minimising wastes and energy-consumption while enhancing purity of the final product, is the corner stone (Anastas, 1998). In this regard, the use of microwaves as a non-conventional heating method has progressively gained attention due to commonly observed acceleration in reactions rates and improved (regio- and chemo-) selectivities and yields in synthetic organic transformations (Kappe, 2004; Caddick, 2009). The claimed cleaner reaction profiles of microwave-assisted processes have thus rapidly projected this kind of heating as a popular method in chemistry, which often replaces the "classical" heating ones. Numerous organic reactions are nowadays fully depicted in the peer-reviewed literature and books. They concern typical synthetic organic approaches (substitutions, alkylations, cycloadditions, esterifications, cyclisations, etc.), organometallic reactions, oxidations and reductions, or polymerisation reactions (Bogdal, 2006).

\section{Scope of this contribution}

Milder reaction conditions, associated to reduced run times (from several hours to a few minutes) and improved yields and selectivities, are the key advantages usually reported for 
microwave-promoted reactions. Even if widely employed in common organic chemistry, microwaves find however fewer applications in carbohydrate chemistry (Corsaro, 2004; Cioffi, 2008; Richel, 2011a). This fact, usually associated to a high number of unwanted and uncontrolled typical sugars reactions such as carbonisation or browning processes, has been overcame by the design of novel sophisticated microwave reactors. Advantageously, microwaves provide nowadays, with remarkable atom efficiency and yields, novel carbohydrate-based chemicals that are not easily obtainable by any other "classical" means or only using painstaking multi-step and energy-consuming protocols (Richel, 2011b). This chapter describes thus the use of microwave processes to mediate key reactions in the field of carbohydrate chemistry. Some examples of benchmark reactions (glycosylations, hydroxyl groups' protection, etc.) under microwave conditions are displayed and highlight the benefits of this microwave approach in terms of yields, selectivities and environmental impact.

\section{Fundamental of microwave technology}

The pioneering works in the microwave-assisted chemistry are attributed to Gedye and Giguere using household microwave ovens (Gedye, 1986; Giguere, 1986). From a theoretical point of view, microwave heating originates from interactions between a given material (reagent, solvent or catalyst) and the electric component of the electromagnetic wave through a dipolar polarisation and/or ionic conduction mechanism. Polar substances like DMSO, ethylene glycol or ethanol are thus suited candidates for microwave applications by opposition to benzene, dioxane or carbon tetrachloride which do not possess permanent dipolar moment. It is recognised that accelerations of reactions rates under microwave conditions are to correlate with a thermal/kinetic effect. Compared to analogous heating under classical conditions (using an oil bath for instance), microwave ensures a fast, selective and homogeneous heating of the reaction vessel (Fig. 1). Convection currents and temperature gradients from vessel walls to the core of the reaction medium, commonly observed using classical heating options, are thus avoided under microwave conditions.

(a)

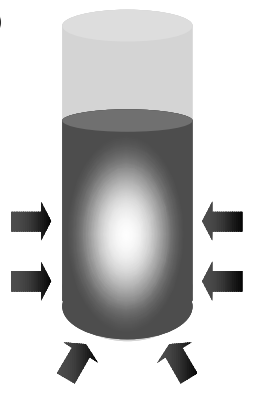

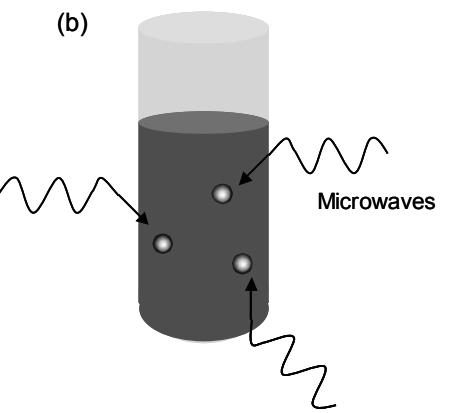

Figure 1. Difference between conventional heating (a) and microwave-assisted heating (b). In (a), heating is provided by thermal conduction. In (b), solvent and/or reagents absorb microwave heating and convert this energy into calorific components. 
The ability of a given substance to convert electromagnetic energy into calorific heat, at specific frequency and temperature, is determined by the loss tangent factor ( $\tan \delta$ ). This factor is described as the ratio between dielectric loss $\left(\varepsilon^{\prime \prime}\right)$ and dielectric constant $\left(\varepsilon^{\prime}\right)$. Practically, substances with high $\tan \delta$ values are required to ensure a convenient and fast microwave heating. By convention, substances are thus categorised into good $(\tan \delta>0.5)$, medium $(0.5>\tan \delta>0.1)$ and poor $(\tan \delta<0.1)$ candidates for microwave $(\mathrm{MW})$ applications. At the labscale, MW is conveniently applied to reactions performed in the liquid state in the presence of a solvent with a high tan $\delta$. However, in order to provide a truly eco-friendly process, syntheses without solvent or in dry-media conditions are also described with reactants and/or catalysts impregnated on a solid microwave-absorbing support such as alumina or silica (Polshettiwar, 2008). In the field of carbohydrate chemistry, reactions under microwave (MW) assistance are usually achieved with saccharides in suspension or in solution in solvent and/or liquid reagents. Highly secured MW reactors, operating in batch or continuous flow conditions, are nowadays available for chemistry. They operate typically at $2.45 \mathrm{GHz}$ and allow an accurate control of the temperature within the MW reaction vessel with an adjustable power, avoiding thus browning. Reactions can also be performed under "closed vessel conditions", at temperatures exceeding the boiling point of the reaction medium.

\section{Chemical transformations of carbohydrates under microwave conditions}

Saccharides, which contain several hydroxyl groups, are polar molecules particularly suited for MW applications. Typical MW-assisted transformations reported in the literature include either reactions involving hydroxyl groups for the production of novel entities (category 1) or dehydration reactions leading to the formation of furfural and related platform molecules (category 2).

\subsection{Microwave-assisted transformations involving hydroxyl groups (category 1)}

Most common reactions of the first category include protections/deprotections of hydroxyl groups, glycosylations, glycosamines formation and halogenations. These reactions are usually catalysed by chemical entities, even if enzymes are proved to offer convenient results for specific cases under microwave conditions. The several hydroxylated stereogenic centres in carbohydrates are difficult to chemically differentiate. Thus, the regioselective derivatisation of such compounds is a challenging task and requires multi-step protocols and purifications. Microwave heating has demonstrated to be a convenient solution, allowing an accurate control of the regioselectivity and the anomeric selectivity by an appropriate tuning of operating conditions. Some relevant examples are proposed herein.

\subsubsection{Hydroxyls protections/deprotections}

Several methods and protocols have been reported for the MW-assisted regioselective protection of hydroxyls, especially in mono- and disaccharides (Corsaro, 2004). They include 
mainly acetal formation with aldehydes and acylation with acetic anhydride or an acyl chloride. All these reactions are promoted using (homogeneous or heterogeneous) acids or bases (Söderberg, 2001) or enzymes (Chen, 2001) in variable solvents, reagents concentrations and operating conditions (temperature and microwave exposure time). The total or regioselective protection (and subsequent deprotection) of specific hydroxyl groups is necessary for the synthesis of building blocks suited for the production of drugs, biologically active materials, and novel high-added value materials. Particularly, acetylation reactions are often the prerequisite step in the synthesis of such complex carbohydrates (Fig. 2). The conventional per-O-acetylation of saccharides is mostly achieved using anhydride acetic as both reagent and solvent. Although this step is high-yielding, long reaction times (several hours) are required and noxious pyridine is mandatory to promote the reaction. Microwave dielectric heating has thus appeared as a more eco-friendly option. Indeed, replacement of pyridine by catalytic amounts of non-toxic sodium acetate or Lewis acid such as zinc chloride is found practically quantitative after only a few minutes of MW heating (Limousin, 1997). With $\mathrm{ZnCl}_{2}$, an equimolar mixture of $\alpha$ and $\beta$ pentaacetates is obtained under $\mathrm{MW}$ conditions, whilst a $\alpha / \beta$ ratio of $7 / 3$ is recovered under oil bath conventional heating (Fig. 2a). Microwave heating affords also convenient results for the complete acetylation of totally $O$ unprotected mono- and disaccharides in $90 \mathrm{sec}$ at 720 Watt under closed vessel conditions using indium(III) chloride catalyst. Reactions performed in acetonitrile with stoichiometric amounts of anhydride acetic are quantitative and affords predominantly the $\alpha$-peracetylated form (Das, 2005). Partial acetylation of carbohydrates is usually achieved with acetyl chloride and pyridine. With 1,2:5,6-di-O-isopropylidene- $\alpha$-D-glucofuranose as the starting material, Söderberg demonstrates that the synergic combination of microwaves and polystyrenesupported base catalysts offers a convenient strategy to obtain in $85 \%$ yield the expected mono-acetylated analogous without formation of side-products (Fig. 2b). This MW approach provides an eco-friendly and time-saving option as the catalyst can be recovered by filtration at the end of the process, thus minimising wastes and purification (Söderberg, 2001).

(a)

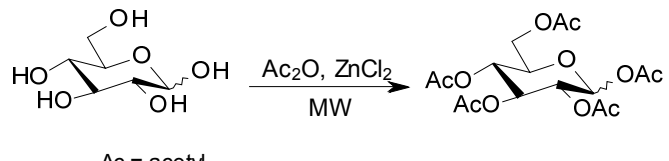

(b)

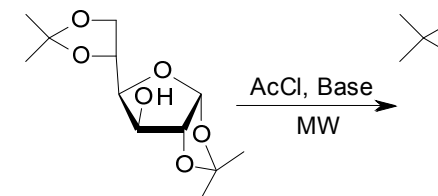

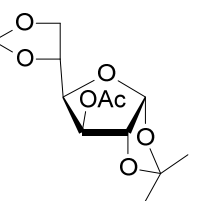

$6.5 \min , 125^{\circ} \mathrm{C}: 98 \%$ yield (83\% under classical heating)

$15 \mathrm{~min}, 180^{\circ} \mathrm{C}: 85 \%$ yield ( $81 \%$ under classical heating after several hours)

Figure 2. Peracetylation (a) and acetylation (b) of selected carbohydrates under MW conditions.

More recently, Witschi et al. describe a novel procedure for the selective acetylation of hydroxyls groups. It relies on a protecting group exchange strategy under microwave assistance starting from per-O-trimethylsilylated pyranosides (Fig. 3). Interestingly, whilst 
the 1,6-O-diacetate monosaccharide is formed under MW exposure, the 6-O-monoacetate adduct is preferentially formed under classical oil bath heating conditions. Reactions go to completion when conducted using acetic acid as a catalyst in neat anhydride acetic for $3 \times 25$ min under MW. For per-O-TMS-galactoside, a 52\% yield in diacetylated adduct is recovered using 2 equivalents of acetic acid. When the galactoside is stirred for 2 days with 2 equiv of acid, the reaction shows a $50 \%$ completion with yields after column chromatography purification reaching 35\% of 6-O-monoacetate (Witschi, 2010).

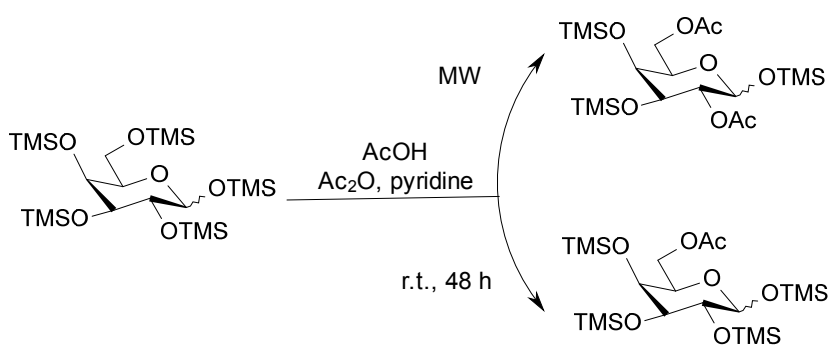

Figure 3. MW-assisted selective acetylation of per-O-TMS-galactoside using protecting group exchange strategy.

Regioselective benzoylation and pivaloylation of carbohydrates is another major item for free hydroxyls protection. Typically performed using benzoyl chloride and pivaloyl chloride, these reactions are only viable when using a base catalyst. Due to the steric bulk of the pivaloyl groups, the protection of hydroxyls through formation of pivaloyl esters is very slow under conventional heating method and requires several hours. Substantial reduction in reaction times (10-20 $\mathrm{min}$ ) is however encountered under MW conditions. Pivaloylation of 1,2:5,6-di-Oisopropylidene- $\alpha$-D-glucofuranose, catalysed by pyridine, provides thus the corresponding 3$\mathrm{O}$-protected target in $68 \%$ yield after $10 \mathrm{~min}$ at $160^{\circ} \mathrm{C}$ in a microwave reactor (Fig. 4a). However, presence of a side-product is recovered after MW exposure as a result of 5,6- to 3,6acetal migration prior to acylation. The formation of this unwanted structure is repressed when using supported polystyrene-bases, i.e. $N, N$-(diisopropyl)aminoethylpolystyrene (PSDIEA) and $N$-(methylpolystyrene)-4-(methylamino)pyridine (PS-DMAP), as alternatives to pyridine. 79 and $88 \%$ yields are thus detected after $15-20 \mathrm{~min}$ at $160-180^{\circ} \mathrm{C}$ in the microwave cavity, whilst only $88 \%$ yield is obtained after $300 \mathrm{~min}$ at room temperature (Söderberg, 2001).

The regioselective benzoylation of carbohydrates affords convenient results using dibutyltin oxide mediators under microwave exposure (Herradón, 1995). Intermediate formation of dibutylstannylene acetal allows hydroxyl regioselective benzoylation after less than $10 \mathrm{~min}$ of microwave exposure with a 500 Watt output (Fig. 4b). This MW methodology is more advantageous than its "classical" analogous involving heating, for several hours, at reflux of a toluene suspension of saccharides and dibutyltin oxide with azeotropic removal of water. Tuning of operation conditions, including both the solvent and the MW power output, modulates the selectivity of dibutylstannylene acetal-mediated benzoylation of representative carbohydrates, such as $\alpha$-D-mannopyranoside (Fig. 5). 
(a)

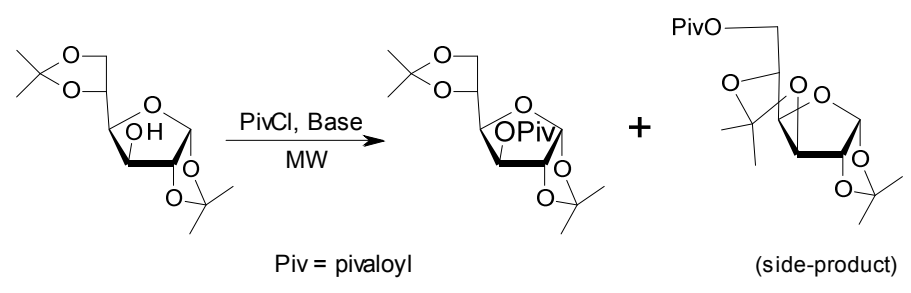

(b)

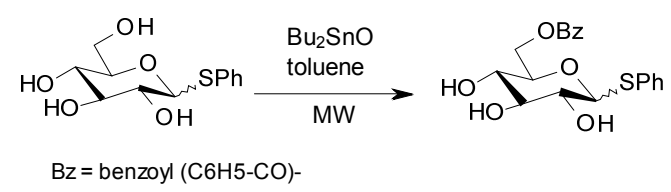

Figure 4. MW-assisted pivaloylation (a) and benzoylation (b).

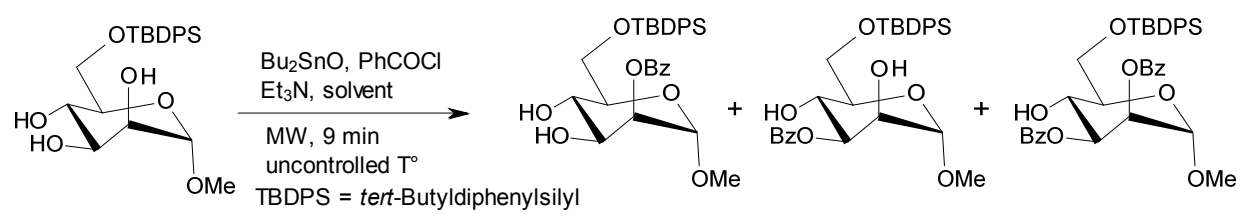

Toluene, maximum MW power output Toluene, minimum MW power output Acetonitrile, minimum MW power output

$\begin{array}{ll}\text { Yield: } & 35 \% \\ & 6 \% \\ & 0 \%\end{array}$

$\begin{array}{ll}25 \% & 10 \% \\ 33 \% & 0 \% \\ 41 \% & 0 \%\end{array}$

Figure 5. MW-promoted benzoylation of $\alpha$-D-mannopyranoside with a tin catalyst.

For carbohydrates not stable in acid conditions, such as 4,6-O-benzylidene- $\alpha$-D-glucopyranose, benzoylation can be achieved via transesterification with methyl benzoate using a week and non nucleophilic base promoter like potassium carbonate and a phase transfer agent (Limousin, 1998). MW permits again a tuning of the regioselectivity. Indeed, after $15 \mathrm{~min}$ in a domestic microwave oven, a $82 \%$ yield of di-benzoylated adduct is recovered, whilst classical oil bath heating affords preferentially the monobenzoylated structures (Fig. 6).

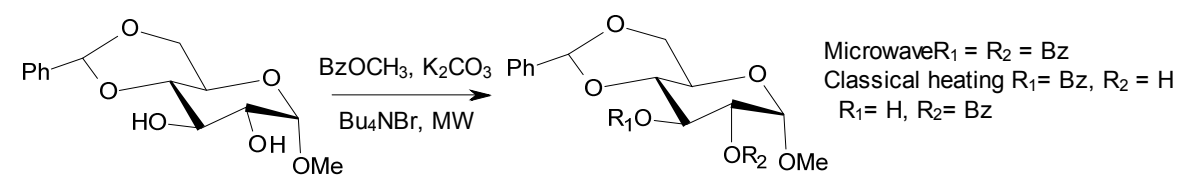

Figure 6. Competition between mono- and dibenzoylation with base catalyst.

Due to the sensitivity of some carbohydrate derivatives at elevated temperatures, microwave methodology suffers however from several limitations, notably for hydroxyls protection processes through acetal formation. As an illustration, the mono-acetalation of $O$ unprotected sucrose in the presence of $p$-toluenesulfonic acid as the catalyst is reported by Salanski and Queneau (Fig. 7). Using citral dimethylacetal, transacetalation provides, under 
classical heating conditions, $83 \%$ yield of a mixture of geranial and neral sucrose acetals ( $E$ and $\mathrm{Z}$ isomers of citral acetals) after $2 \mathrm{~min}$ at $100^{\circ} \mathrm{C}$. When applying microwave as the heating source, yield drops to $42 \%$ after an identical runtime in open vessel conditions. Cleavage of the glycosidic linkage is denoted and leads also to the formation of unwanted side-products (17-26\% yield after 2 to $10 \mathrm{~min}$ of MW exposure) (Salanski, 1998).

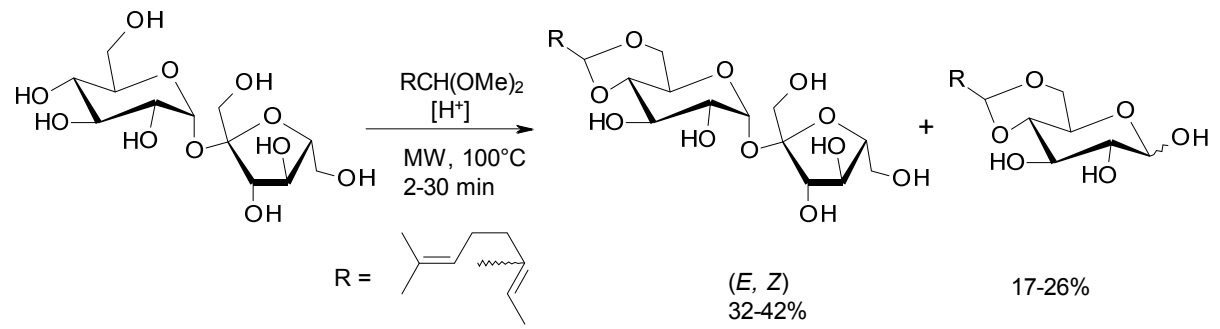

Figure 7. Protection of sucrose through acetal formation.

For deprotection processes, microwave heating is decisive and powerful, as a drastic diminution of reaction times is observed without affecting the initial anomeric configuration (Corsaro, 2004). An efficient and green protocol concerns the use of neutral alumina to ensure the regioselective depivaloylation of a set of carbohydrate derivatives (Fig 8a). Whilst the depivaloylation does not occur using a conventional heating source, the regioselective deprotection of primary hydroxyl functions is observed after a few minutes à $75^{\circ} \mathrm{C}$ under MW assistance (Ley, 1993). The fast, efficient and clean deacetalation of several carbohydrate 4,6-di-O-benzylidene acetals is also reported using silica supported reagents under microwave irradiation in solvent-free conditions (Fig. 8b). Yields are superior to $80 \%$ after $7 \mathrm{~min}$ in a domestic oven at 500 Watt, without anomerisation effect (Couri, 2005).

(a)
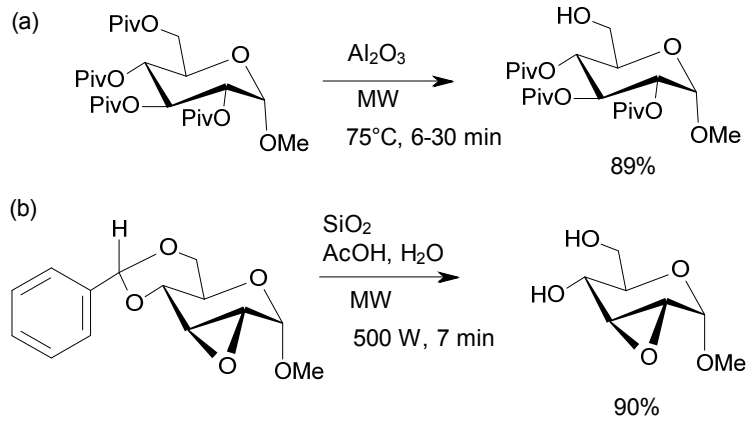

Figure 8. MW-assisted deprotection initiatives: deacylation (a) and deacetalation (b).

\subsubsection{O-glycosylations}

O-glycosylation consists in the specific derivatisation of the anomeric hydroxyl position in carbohydrate structures. This reaction is one of the most emblematic in carbohydrate 
chemistry and is extensively reviewed (Demchenko, 2008). Several parameters are found to influence the (stereoselective) formation of glycosyl linkages such as solvent, catalyst, additives, nature of both leaving groups and surrounding protecting groups. In particular, the formation of alkyl- and aryl-glycosides has find special attention in both academic and industrial worlds, due to the importance of these molecules as non-toxic and biodegradable surfactants, liquid crystals, and pharmaceutical agents (Razafindralambo 2011 \& 2012). Under microwave conditions, the introduction of alkyl substituents on the $\mathrm{OH} 1$ position is the most extensively developed, notably for the large-scale production of surface-active agents, and relies on the reaction of an alkyl alcohol with unprotected saccharide (Fischertype glycosylation) or with completely O-protected carbohydrate compounds (Helferichtype glycosylation). These glycosylations are catalysed either by chemical promoters or by enzymes. Alkylation of D-glucose by fatty alcohol using acid catalysts is the oldest reaction reported under MW conditions (Fig. 9). Fatty alcohol advantageously plays both the role of solvent and reagent, even if glucose is insoluble in most alcohols. After 10 min of MW exposure with a 200 Watt maximum output, a $15 \%$ yield in glycosylated adduct is obtained using homogeneous Brönsted (or Lewis) acids or heterogeneous promoters (zeolites, ion exchange resins, montmorillonites). No control of the temperature is provided using this domestic MW equipment. Such a low yield is to correlate with unwanted glycoside decomposition under acidic conditions during the run and to browning reactions (Limousin, 1997). This result is not completely satisfactory but remains quite noteworthy compared to other classical protocols. The versatility of this MW Fischer glycosylation is now published for various carbohydrates, including mostly easily available monosaccharides like Dmannose, D-galactose, $\mathrm{N}$-acetyl-D-glucosamine and $\mathrm{N}$-acetyl-D-galactosamine. Glycosylation of representative long chain alcohols is efficient with acidic ion-exchange resins between 90 and $120^{\circ} \mathrm{C}$. Typically, the reaction goes to completion after less than $2 \mathrm{~min}$ of MW heating, compared with $4 \mathrm{~h}$ at reflux, and affords preferentially the thermodynamic $\alpha$ adduct. The anomeric distribution seems quite identical under MW and conventional oil bath heating conditions (Bornaghi, 2005). A valuable scaling-up of this Fischer-type glycosylation is proposed by Nüchter up to the kg-scale with an efficient improvement of economic efficiency, using an appropriated commercial MW batch reactor (Nüchter, 2001). An accurate control of the temperature inside the reactor cavity provides a good quality result. Practically, monosaccharide (glucose, mannose or galactose) reacts with a 3-30-fold excess of alcohol (methanol, ethanol, butanol or octanol) in the presence of sulphuric acid catalyst. Nearly quantitative yields are recovered after $20-60 \mathrm{~min}$ of $\mathrm{MW}$ heating at $60-140^{\circ} \mathrm{C}$, as a function of the nature of the alcohol. Further assays, using continuous flow methods, afford quantitative yields with a MW chamber heated at $110-140^{\circ} \mathrm{C}$. More recent work highlights the use of Brönsted acids immobilised on silica as convenient solid catalysts with advantages in manufacturing scale synthesis (Fig. 9). The Fischer-type glycosylation of Dglucose with $n$-decanol in solvent-less conditions leads to the expected adduct in $82 \%$ yield as an equimolar mixture of $\alpha$ and $\beta$ anomers (Richel, 2011b). This protocol offers workup, economic and environmental advantages as catalyst can be recovered by filtration and reused. 

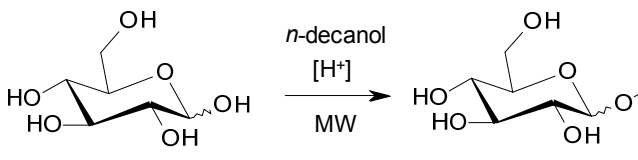

$\alpha, \beta$

Figure 9. Fischer-type glycosylation of D-glucose with $n$-decanol under MW conditions for the production of a potent surface-active agent.

An alternative three-step protocol (complete acylation-glycosylation-deprotection) is proposed under MW. Due to the increased thermal stability of acylated compounds, MW Helferich-type reactions are expected to be more effective (Fig. 10). Indeed, addition of decanol on per-Oacetylated glucose offers a $74 \%$ yield (mainly the $\alpha$-pyranose form) after a running time of 3 min at $113^{\circ} \mathrm{C}$ using zinc chloride as the catalyst. Extended MW exposure times have a detrimental effect over the yield ( $25 \%$ after $300 \mathrm{~min}$ ) due to decomposition of decyl glycoside. No rationalisation between the furanose-pyranose (and anomeric) distribution and processing conditions is found at this stage (Limousin, 1997). The first elements of answer are proposed by Kovensky and Ferlin in 2008. A microwave temperature of $115^{\circ} \mathrm{C}$ is found as a good compromise to obtain high glycosylation rates and low decomposition products contents. Both yields and anomeric distributions are dependent on the boiling/melting points and the polarity of the selected alkyl alcohol. Polar alcohols with low boiling/melting points lead to the formation of the corresponding thermodynamic $\alpha$ adduct, while long chain alcohols provide preferentially the kinetic $\beta$ anomer in less than $5 \mathrm{~min}$. For each alcohol, an optimum reaction time, beyond which the formed alkyl glycoside is deteriorated, is estimated (Ferlin, 2008).

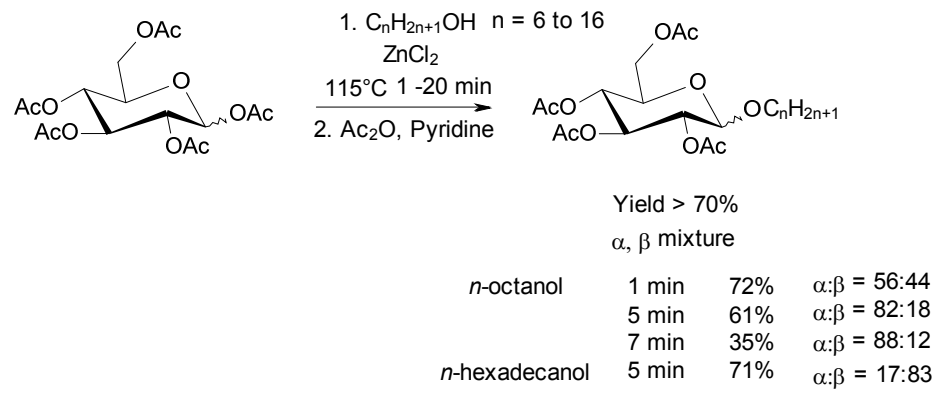

Figure 10. Helferich-type glycosylation of per-O-acetylated glucose with alkyl alcohols. Influence of the chain length on both yields and $\alpha / \beta$ ratio.

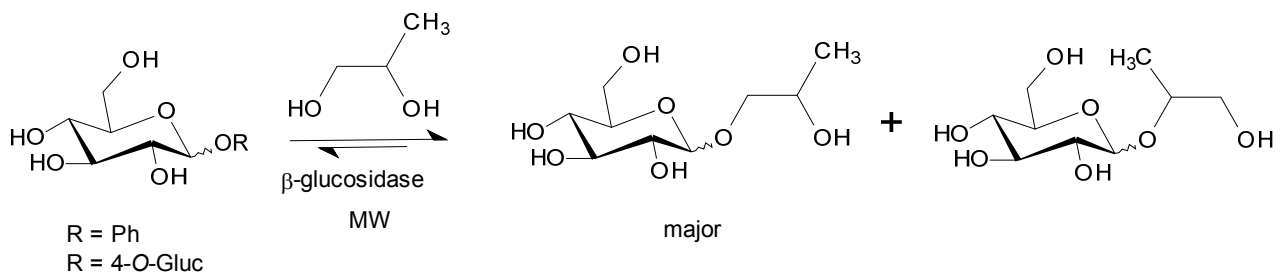

Figure 11. MW-assisted trans-glycosylation of several carbohydrates acceptors and propane-1,2-diol. 
Enzymatic methods under microwave conditions are also explored as convenient option for glycosylations. Using $\beta$-glucosidase, the synthesis of several glycosides by trans-glycosylation is reported with either phenyl $\beta$-D-glucoside or cellobiose as donors and propane-1,2-diol as acceptor (Fig. 11). The process is performed in dry-media conditions, with reagents adsorbed on alumina in an open vessel system. This microwave reaction, achieved at controlled temperature (between 80 and $110^{\circ} \mathrm{C}$ ), leads to noticeable advantages, in terms of yields and selectivity, when compared to classical heating. In particular, trans-glycosylations go to completion after less than $2 \mathrm{~h}$, while hydrolysis is lowered to $10 \%$ (Gelo-Pujic, 1997).

(a)

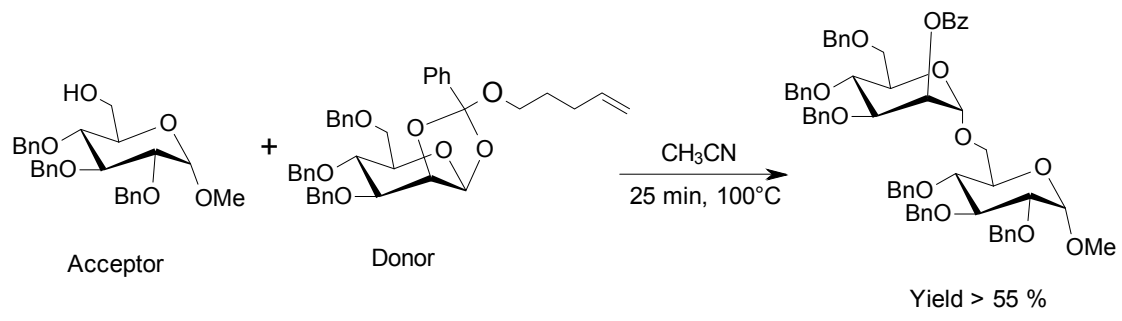

(b)
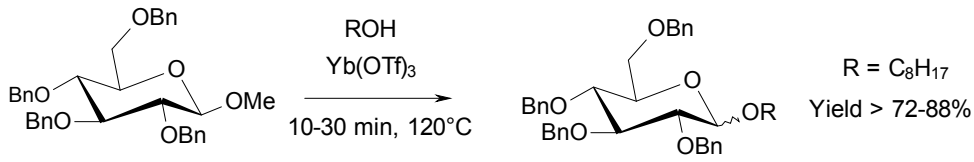

(c)
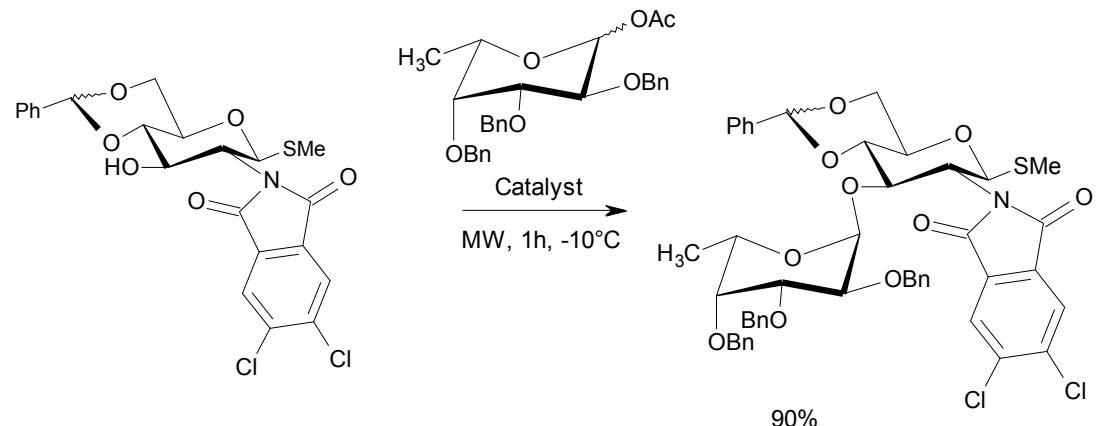

$90 \%$

(d)
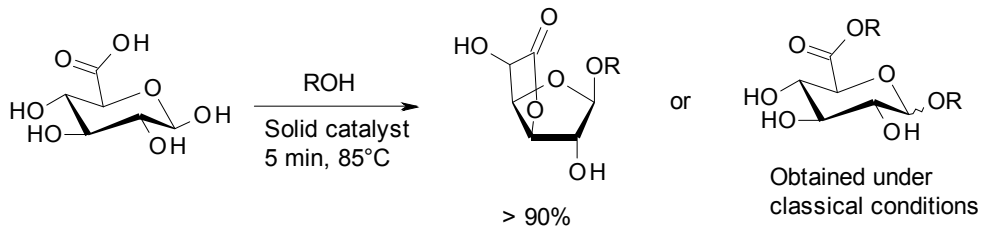

$\left.\mathrm{R}=\mathrm{Me},\left(\mathrm{CH}_{2}\right)_{3} \mathrm{CH}_{3}, \mathrm{CH}_{2}\right)_{7} \mathrm{CH}_{3}, \mathrm{Ph}$

$\left(\mathrm{CH}_{2}\right) \mathrm{Cl},\left(\mathrm{CH}_{2}\right)_{2} \mathrm{CH}=\mathrm{CH}_{2},\left(\mathrm{CH}_{2}\right)_{4} \mathrm{CH}=\mathrm{CH}_{2}$

Figure 12. Novel microwave-assisted glycosylations. 
Beside the benchmark Fischer- and Helferich-glycosylations, novel strategies under microwave heating are nowadays emerging in the peer-reviewed literature. Some examples are proposed in Fig. 12. Most of them concern the catalytic conjugation of alcohols (designated as acceptors) to protected 4-pentenyl glycosides or methyl glycosides (donors), notably for the production of di- and oligosaccharides. As an illustration, the synthesis of disaccharides is described in acetonitrile starting from 4-pentenyl donors and is achieved in high yields after less than $25 \mathrm{~min}$ at $100^{\circ} \mathrm{C}$ in closed vessel conditions without side-products formation (Fig. 12a) (Mathew, 2004). The use of more effective Lewis acids, like ytterbium(III) triflate, affords high yields for the anchorage of long alkyl chains on benzoylated glucose derivatives (Fig 12b). This acetal-exhange type glycosylation is performed in dichloromethane below $100^{\circ} \mathrm{C}$ in closed vessel conditions. This constitutes the main advantage of microwave over conventional heating techniques (Yoshimura, 2005). Application of very low temperatures $\left(-10^{\circ} \mathrm{C}\right)$ within the MW cavity, attained by simultaneous cooling, offers a convenient approach for the synthesis of valuable saccharides and Lewis $\mathrm{X}$ oligosaccharides (Fig. 12c). The process involves a fucosyl acetate donor and a glucosamine acceptor in about $2 \mathrm{~h}$ in ether. Yields culminate at about $80-90 \%$ without side-products formation, whilst the expected disaccharide is not obtained using classical protocols (Shimizu, 2008). Another strategy demonstrates the usefulness of microwave heating, in synergy with heterogeneous acid catalysts, for the regioselective functionalisation of D-glucuronic acid. Conversions are quantitative when performed in solvent-less conditions with an excess of alcohol under microwave exposure. Less than $10 \mathrm{~min}$ of heating at $85^{\circ} \mathrm{C}$ in closed vessel conditions affords selectively the corresponding $\beta$-glucurono-6,3-lactone adduct. When heating in an oil bath, only the disubstituted adducts, as a mixture of $\alpha, \beta$-furanoses and $\alpha, \beta$-pyranoses, are formed through competitive esterification and O-glycosylation processes (Fig. 12d). Comparative yields are recorded after 6 to $24 \mathrm{~h}$ at $85^{\circ} \mathrm{C}$, evidencing that MW accelerates reactions and imparts over the selectivity. This methodology is eco-friendly as water in the only by-product and the solid catalyst can be reused for consecutive batch MW runs (Richel, 2012). These monosubstituted glucuronolactones are not easily attainable using other conventional protocols and required multi-step synthesis. These original products find special relevance in the field of surface-active agents and emulsifiers (Razafindralambo, 2011).

\subsubsection{Glycosamines synthesis}

Glycosamines are valuable compounds as glyco-amino acid building blocks for glycopeptides synthesis. Their preparation is traditionally achieved using a Kochetkov reductive amination procedure. Practically, a treatment at room temperature for 6 days of saccharides in aqueous solution with 50 equiv of ammonium bicarbonate affords the expected $\beta$-glycosaminated adducts in high yields. Another route involves the heating at $42^{\circ} \mathrm{C}$ for $36 \mathrm{~h}$ of mono- or disaccharides with an aqueous solution of ammonia in the presence of one equivalent of ammonium hydrogen carbonate. However, $\beta$-glycosamines can be deteriorated under aqueous solutions, even with accurate $\mathrm{pH}$ control. Nowadays, the preparation of these glycosamines is typically performed under microwave conditions, at $40^{\circ} \mathrm{C}$ for $90 \mathrm{~min}$, by selective amination of reductive carbohydrates using reduced quantities 
of ammonium bicarbonate (5 equiv) (Fig. 13). Under closed vessels conditions, the reaction is achieved in methanol or in anhydrous dimethylsulfoxide to prevent degradation and side-products formation (Liu, 2010). Yields are of about 70-95\%.

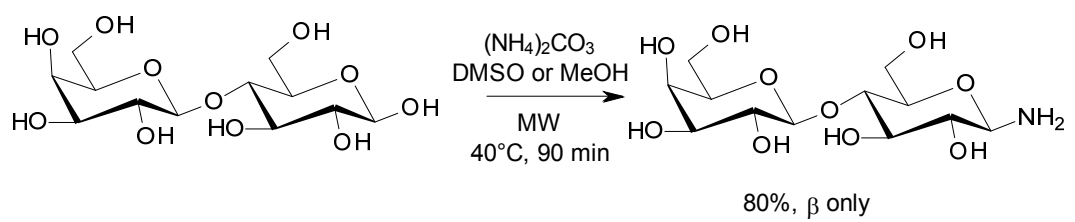

Figure 13. Synthesis of 1-amino-1-deoxy- $\beta$-D-lactoside by microwave-assisted Kochetkov amination of unprotected lactose.

In summary, reactions of the first category, involving hydroxyls manipulation appear generally faster under MW conditions than their "classical" counterparts. Expected products are indeed obtained in good yields after only a few minutes. The use of eco-friendly and non-toxic (heterogeneous) and milder catalysts in synergy with microwaves, affords ideal "green chemical" conditions and minimise workup. Microwave allows also the formation of specific protected products, not easily accessible under conventional "heating" conditions.

\subsection{Microwave-assisted production of platform chemicals (category 2)}

The production of 5-HMF (5-hydroxymethylfurfural), a five-membered ring compounds ranging in the US Top 10 of most valuable chemicals issued from biomass, is currently extensively investigated worldwide. Most research concerns its production via thermallyinduced acid-catalysed dehydration of fructose, typically in dimethylsulfoxide, in the presence of homogeneous acid catalysts $\left(\mathrm{HCl}_{1} \mathrm{H}_{2} \mathrm{SO}_{4}, \mathrm{H}_{3} \mathrm{PO}_{4}\right.$, oxalic or levulinic acids) (Fig. 14). Reactions produces selectively $40-60 \%$ yields of 5-HMF with complete conversion of starting sugar materials but suffers from drawbacks in terms of acids recovery and equipment corrosion. Heterogeneous catalysts offer workup and environmental advantages associated to high 5-HMF selectivity but provide low fructose conversions (30-60\% only after up to $2 \mathrm{~h}$ ). Microwave has progressively emerged as an attractive heating source allowing energy savings and improved yields and selectivity. Recent developments in the field have been reviewed by Richel in 2011 (Richel, 2011b). They include the use of heterogeneous catalysts in various media (organic solvents, water, ionic liquids or biphasic systems).

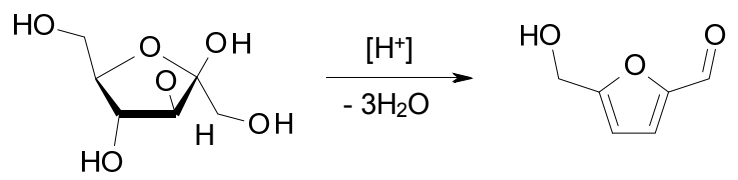

Figure 14. Catalytic dehydration of fructose for the production of 5-HMF.

Titanium and zirconium oxides, as sulphated zirconia, are effective catalysts for this dehydration, especially under microwave conditions. Yields in 5-HMF reach 35\% for a fructose conversion of $78 \%$ after only 5 min heating at $200^{\circ} \mathrm{C}$ in closed vessels conditions 
("microwave hot compressed water process"), while 5-HMF yield and fructose conversion are respectively of 12 and $27 \%$ after an identical runtime using a sand bath heating. Zirconium oxide demonstrates great performances for the catalytic dehydration of glucose, favouring isomerisation of glucose to fructose prior dehydration (Qi, 2009). When using an acid ion-exchange resin in an acetone/dimethylsulfoxide system, improvement in 5-HMF selectivity is encountered together with high yields (98\% fructose conversion with 5-HMF selectivity of $92 \%$ for $10 \mathrm{~min} \mathrm{MW}$ assisted reaction at $150^{\circ} \mathrm{C}$ in closed vessel conditions). Addition of low-boiling point acetone improves products separation and reduces adverse environmental impacts. The same results are published for strong ion-exchange resin in acetone-water systems, providing a truly eco-friendly process (Qi, 2008b). The common Lewis acid catalyst, aluminium(III) chloride, promotes also efficiently the dehydration of carbohydrate substrates, in water-organic solvents and in water. 5-HMF yield is higher when using an organic solvent than in pure water. Involvement of ionic liquids as convenient media for the conversion of D-glucose in 5-HMF is effective using chromium(III) chloride as a catalyst. With microwave heating at $140^{\circ} \mathrm{C}$ for $30 \mathrm{sec}$, a $5-\mathrm{HMF}$ yield of $71 \%$ is obtained for 96\% glucose in 1-butyl-3-methyl imidazolium chloride as the solvent (Qi, 2010). Lignin-based solid catalyst, produced by carbonisation and sulfonation of raw lignocellulosic materials, seems nowadays to be the most active catalyst for the dehydration of carbohydrates. In synergy with MW, 84\% 5-HMF yield is obtained with $98 \%$ fructose conversion rate at $110^{\circ} \mathrm{C}$ for $10^{\circ} \mathrm{C}$. A mixture of dimethylsulfoxide-ionic liquid has a beneficial effect over the selectivity of the process. Interestingly, the catalyst can be recovered after the reaction and reused for consecutive batch reactions (Guo, 2012).

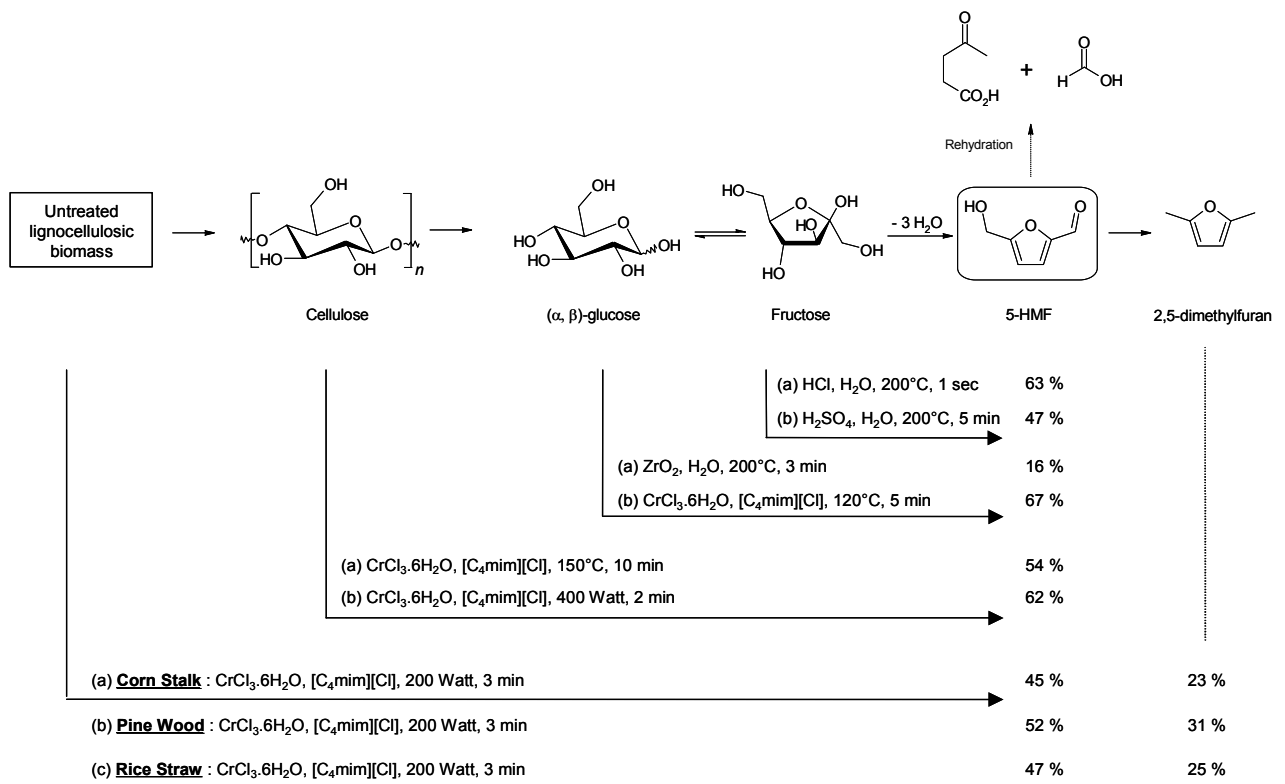

Figure 15. Overview of MW-assisted production of 5-HMF from lignocellulosic biomass materials. 
Scaling up of the MW-assisted fructose dehydration process has been recently evaluated using a stop-flow MW reactor system and compared to a continuous cartridge-based reactor. Results highlight that 5-HMF yield can attain $86 \%$ yield after $2 \mathrm{~min}$ of MW exposure (91\% using a cartridge-base reactor) with a 5-HMF productivity of $0.72 \mathrm{~g} / \mathrm{h}$ (versus $2.07 \mathrm{~g} / \mathrm{h}$ for the continuous process). Even if quite less productive, the MW-assisted methodology is more selective and cleaner as no side-product is detected in the final mixture (Schoen, 2011).

Besides these aforementioned benchmark reactions, research has shifted toward the exploration of other carbohydrates or crude lignocellulosic feedstocks as raw materials. Some relevant illustrations are reported in Fig. 15. They concern the use of ionic liquids as solvent. For environmental concern, water is also envisioned with homogeneous Brönsted acids or metal chloride catalysts (Dutta, 2012). The production of 2-furfural, another platform chemicals, receives progressively interest. Its MW-assisted production from xylose, xylan and wheat straw is highly efficient in the presence of hydrochloric acid. A $48 \%$ furfural yield is obtained from wheat straw after $1 \mathrm{~min}$ of microwave heating between 140 and $190^{\circ} \mathrm{C}$. (Yemis, 2011).

\section{Conclusion}

In the present chapter, we contribute to illustrate some relevant carbohydrate reactions under microwave heating. This heating mode appears as efficient for several key reactions like hydroxyls protection and deprotection and O-glycosylations. The production of 5-HMF from mono-/di-/polysaccharides is another special subject in MW science. Improvement of both yields and (regio- and anomeric) selectivities is generally reported together with a decrease in reaction times. MW offers also advantages as energy-saving and workup facilities in agreement with the green chemistry concept.

\section{Author details}

Aurore Richel and Michel Paquot

University of Liege, Belgium

\section{Acknowledgement}

This work was carried out in the framework of the "Technose" Excellence Programme (project number 716757) supported by the "Région Wallonne" (Belgium). The authors are grateful to the "Région Wallonne" for its financial support.

\section{References}

Anastas, P. T.; Warner, J. C. (1998). Green Chemistry: Theory and Practice; Oxford University Press: Oxford

Bogdal, D. (2006). Microwave-assisted synthesis. One hundred reaction procedures. Tetrahedron Organic Chemistry Series, Vol. 25; Elsevier.

Bornaghi, L. F.; Poulsen, S. A. (2005). 
Bozell, J. J.; Petersen, G. R. (2010). Technology development for the production of biobased products from biorefinery carbohydrates-the US Department of Energy's "Top 10" revisited. Green Chem., 12, 539-554

Caddick, S.; Fitzmaurice, R. (2009). Microwave enhanced synthesis. Tetrahedron, 65, 3325-3355

Chen, S. T.; Sookkheo, B.; Phutrahul, S.; Wang, K. T. (2001). Enzymes in nonaqueous solvents: Applications in carbohydrate and peptide preparation. Methods in Biotechnology, 15, 373-400.

Cioffi, E. A. (2008). High-energy glycoconjugates: synthetic transformations of carbohydrates using microwave and ultrasonic energy. Curr. Top. Med. Chem., 8, 152-158

Corsaro, A.; Chiacchio, U.; Pistarà, V.; Romeo, G. (2004). Microwave-assisted chemistry of carbohydrates. Curr. Org. Chem., 8, 511-538

Das, S. K.; Reddy, K. A.; Krovvidi, V. L. N. R.; Mukkanti, K. (2005). $\mathrm{InCl}_{3}$ as a powerful catalyst for the acetylation of carbohydrate alcohols under microwave irradiation. Carbohydr. Res., 340, 1387-1392

Demchenko, A. V. (2008). Handbook of Chemical Glycosylation; Advances in Stereoselectivity and Therapeutic Relevance, Wiley-VCH, Weinheim

Dutta, S.; De, S.; Alam, M. I. ; Abu-Omar, M. M. ; Saha, B. (2012). Direct conversion of cellulose and lignocellulosic biomass into chemicals and biofuel with metal chloride catalysts. J. Catal., 288, 8-15

Ferlin, N. ; Duchet, L. ; Kovensky, J. ; Grand, E. (2008). Microwave-assisted synthesis of long-chain alkyl glucopyranosides. Carbohydr. Res., 343, 2819-2821

Gedye, R. ; Smith, F.; Westaway, K.; Ali, H.; Baldisera, L.; Laberge, L.; Roussel, J. (1986). The use of microwave ovens for rapid organic synthesis. Tetrahedron Lett., 27, 279-282

Gelo-Pujic, M.; Guibé-Jampel, E.; Loupy, A.; Trincone, A. (1997). Enzymatic glycosidation in dry media under microwave irradiation. J. Chem. Soc., Perkin Trans. 1, 1001-1002

Giguere, R. J.; Bray, T; L.; Duncan, S. M.; Majetich, G. (1986). Application of commercial microwave ovens to organic synthesis. Tetrahedron Lett., 27, 4945-4948.

Herradón, B.; Morcuende, A.; Valverde, S. (1995). Microwave Accelerated Organic Transformations: Dibutylstannylene Acetal Mediated Selective Acylation of Polyols and Amino Alcohols using Catalytic Amounts of Dibutyltin Oxide. Influence of the Solvent and the Power Output on the Selectivity. Synlett, 455-458

Guo, F.; Fang, Z.; Zhou, T. J. (2012). Conversion of fructose and glucose with lignin-derived

carbonaceous catalyst under microwave irradiation in dimethyl sulfoxide-ionic liquid mixtures. Biores. Technol., 112, 313-318.

Kappe, C. O. (2004). Controlled microwave heating in modern organic synthesis. Angew. Chem. Int. Ed., 43, 6250-6284

Lichtenthaler, F. W.; Peters, S. (2004). Carbohydrates as green raw materials for the chemical industry. C. R. Chimie, 7, 67-90

Limousin, C.; Cléophax, J.; Petit, A.; Loupy, A. ; Lukacs, G. (1997). Solvent-Free Synthesis of Decyl D-Glycopyranosides Under Focused Microwave Irradiation. J. Carbohydr. Chem., 16, 327-342

Limousin, C.; Cléophax, J.; Petit, A.; Loupy, A. (1998). Synthesis of benzoyl and dodecanoyl derivatives from protected carbohydrates under focused microwave irradiation. Tetrahedron, 54, 13567-13578

Liu, X.; Zhang, G.; Chan, K.; Li, J. (2010). Microwave-assisted Kochetkov amination followed by permanent charge derivatization: a facile strategy for glycomics. Chem. Commun., 46, 7424-7426. 
Mathew, F.; Jayaprakash, K. N.; Fraser-Reid, B.; Mathew, J.; Scicinski, J. (2004). Microwave-assisted saccharide coupling with n-pentenyl glycosyl donors. Tetrahedron Lett., 44, 9051-9054.

Nüchter, M.; Ondruschka, B.; Lautenschlarger, W. (2001). Microwave-assisted synthesis of alkyl glycosides. Synth. Commun., 31, 1277-1283

Polshettiwar, V.; Varma, R. S. (2008). Microwave-Assisted Organic Synthesis and Transformations using Benign Reaction Media. Acc. Chem. Res., 41, 629-639.

Qi, X.; Watanabe, M.; Aida, T.; Smith, T. L. (2008). Catalytical conversion of fructose and glucose into 5-hydroxymethylfurfural in hot compressed water by microwave heating. Catal. Commun., 9, 2244-2249.

Qi, X.; Watanabe, M.; Aida, T.; Smith, T. L. (2009). Sulfated zirconia as a solid acid catalyst for the dehydration of fructose to 5-hydroxymethylfurfural. Catal. Commun., 10, 1771-1775

Qi, X.; Watanabe, M.; Aida, T.; Smith, T. L. (2010). Fast transformation of glucose and di/polysaccharides into 5-hydroxymethylfurfural by microwave heating in an ionic liquid/catalyst system. ChemSusChem, 3, 1071-1077

Razafindralambo, H.; Richel, A.; Wathelet, B.; Blecker, C.; Wathelet, J. P.; Brasseur, R.; Lins, L.; Miñones, J.; Paquot, M. (2011). Monolayer Properties of Uronic Acid Bicatenary Derivatives at the Air-Water Interface: Effect of Hydroxyl Group Stereochemistry Evidenced by Experimental and Computational Approaches. Phys. Chem. Chem. Phys. 13, 15291-15298

Razafindralambo, H.; Richel, A.; Paquot, M. Lins, L. ; Blecker, C. (2012). Liquid Crystalline Phases Induced by the Hydroxyl Group Stereochemistry of Amphiphilic Carbohydrate Bicatenary Derivatives. J. Phys. Chem. B, 116, 3998-4005

Richel, A.; Laurent, P.; Wathelet, B.; Wathelet, J. P.; Paquot, M. (2011a). Microwave-assisted conversion of carbohydrates. State of the art and outlook. C. R. Chimie, 14, 224-234.

Richel, A.; Laurent, P.; Wathelet, B.; Wathelet, J. P.; Paquot, M. (2011b). Current perspectives on microwave-enhanced reactions of monosaccharides promoted by heterogeneous catalysts. Catal. Today, 107, 141-147

Richel, A.; Laurent, P.; Wathelet, B.; Wathelet, J. P.; Paquot, M. (2012). Efficient microwavepromoted synthesis of glucuronic and galacturonic acid derivatives using sulfuric acid impregnated on silica. Green Chem. Lett. Rev., 5, 179-186.

Salanski, P., Descotes, G., Bouchu, A., Queneau, Y. (1998). Monoacetalation of unprotected sucrose with citral and ionones. J. Carbohydr. Chem., 17, 129-142

Schoen, M.; Schnuerch, M.; Mihovilovic, M. D. (2011). Application of continuous flow and alternative energy devices for 5-hydroxymethylfurfural production. Mol. Divers. 15, 639-643.

Shimizu, H.; Yoshimura, Y.; Hinou, H.; Nishimura, S.-I. (2008). A novel glycosylation method part 3: study of microwave effects at low temperatures to control reaction pathways and reduce byproducts. Tetrahedron, 2008, 10091-10096

Söderberg, E.; Westman, J.; Oscarson, S. (2001). Rapid carbohydrate protecting group manipulations assisted by microwave dielectric heating. J. Carbohydr. Chem., 20, 397-410.

Witschi, M. A. \& Gervay-Hague' J. G. (2010). Selective Acetylation of per-O-TMS-Protected Monosaccharides. Org. Lett., 12, 4312-4315

Yemis, O.; Mazza, G. (2011). Acid-catalyzed conversion of xylose, xylan and straw into furfural by microwave-assisted reaction. Biores. Technol., 102, 7371-7378.

Yoshimura, Y. ; Shimizu, H. ; Hinou, H. ; Nishimura, S.-I. (2005). A novel glycosylation concept; microwave-assisted acetal-exchange type glycosylations from methyl glycosides as donors. Tetrahedron Lett., 46, 4701-4705 